\title{
Chiral symmetry breaking and monopoles in gauge theories
}

\author{
Adith Ramamurti ${ }^{*}$ and Edward Shuryak ${ }^{\dagger}$ \\ Department of Physics and Astronomy, Stony Brook University, Stony Brook, New York 11794, USA
}

(Received 27 February 2018; published 15 July 2019)

\begin{abstract}
QCD monopoles are magnetically charged quasiparticles whose Bose-Einstein condensation (BEC) at $T<T_{c}$ creates electric confinement and flux tubes. The "magnetic scenario" of QCD proposes that scattering on the noncondensed component of the monopole ensemble at $T>T_{c}$ plays an important role in explaining the properties of strongly coupled quark-gluon plasma (sQGP) near the deconfinement temperature. In this paper, we study the phenomenon of chiral symmetry breaking and its relation to magnetic monopoles. Specifically, we study the eigenvalue spectrum of the Dirac operator in the basis of fermionic zero modes in an SU(2) monopole background. We find that as the temperature approaches the deconfinement temperature $T_{c}$ from above, the eigenvalue spectrum has a finite density at $\omega=0$, indicating the presence of a chiral condensate. In addition, we find the critical scaling of the eigenvalue gap to be consistent with that of the correlation length in the $3 \mathrm{~d}$ Ising model and the BEC transition of monopoles on the lattice.
\end{abstract}

DOI: $10.1103 /$ PhysRevD.100.016007

\section{INTRODUCTION}

The possible existence of magnetic monopoles in electrodynamics fascinated leading physicists in the 19 th century. With the development of quantum mechanics, Dirac famously related the existence of monopoles with electric charge quantization [1]. Monopoles in quantum electrodynamics, however, were never found.

With the advent of non-Abelian gauge theories, classical solitons with magnetic charge were found by 't Hooft [2] and Polyakov [3] in the Georgi-Glashow model. Such monopoles play important role in all other theories with an adjoint scalar field, notably in theories with extended supersymmetry $\mathcal{N}=2,4$.

In theories without such scalars, e.g., pure gauge theories, one can use the same 't Hooft-Polyakov solution with the zeroth component of the gauge field $A_{0}$ acting as the "Higgs"; this option leads to the semiclassical theory of instanton-dyons. For a recent short review, see Ref. [4]. These objects, however, are pseudo-particles and not particles, existing only in the Euclidean formulation of the theory, which severely limits their phenomenological applications. We will not discuss this issue further and only remind the reader that, in the case of extended

\footnotetext{
*adith.ramamurti@stonybrook.edu

†edward.shuryak@stonybrook.edu
}

Published by the American Physical Society under the terms of the Creative Commons Attribution 4.0 International license. Further distribution of this work must maintain attribution to the author(s) and the published article's title, journal citation, and DOI. Funded by SCOAP. supersymmetry, the partition functions in terms of monopoles and instanton-dyons were shown to be equal, related by the so-called Poisson duality [5-7].

In spite of the monopole solution lacking in theories without scalars, Nambu [8], 't Hooft [9], and Mandelstam [10] proposed the "dual superconductor" model of the electric color confinement. In this model, the Bose-Einstein condensation (BEC) of monopoles at $T \leq T_{c}$ expels electric field from the vacuum into confining flux tubes.

In lattice studies of gauge theories, monopoles have been identified via procedures including choosing specific gauges, such as maximal Abelian gauge. Their locations and paths were positively correlated with gauge-invariant observables, such as the action and square of the magnetic field [11]. The monopoles were found to create a magnetic current around the electric flux tube, stabilizing them $[12,13]$. In the Landau gauge, while monopole-type singularities themselves are not present, the physical properties that they source, e.g., the magnetic displacement current, are still present and are gauge invariant [14]. Furthermore, their motion and correlations were shown to be exactly as expected for a Coulomb plasma [15-17]. The deconfinement critical temperature $T_{c}$ does coincide accurately with that of monopole BEC transition $[16,18,19]$, and the BEC transition, along with the magnetic charge, has been shown to be gauge independent [20-22].

The "magnetic scenario" of QCD [17,23,24] assumes the presence of noncondensed monopoles in quark-gluon plasma (QGP). Unlike quarks and gluons, which have vanishing densities at $T \rightarrow T_{c}$, the monopole density has a peak near $T_{c}$ and is dominant there. Monopole-gluon and monopole-quark scattering were shown to play a significant 
role in kinetic properties of QGP, such as the shear viscosity $\eta$ [25] and the jet quenching parameter $\hat{q}$ [26-28]. The noncondensed monopoles should also lead to electric flux tubes at $T>T_{c}$ [24], which indeed were recently observed on the lattice [29]. Thus, there is a growing amount of phenomenological evidence suggesting magnetic monopoles do exist, not only as a confining condensate at $T \leq T_{c}$, but also as noncondensed quasiparticles at $T>T_{c}$.

While the central role of monopoles in the confinementdeconfinement transition was recognized long ago, their relation to another important nonperturbative aspect of QCD-like theories, chiral symmetry breaking, has attracted much less attention. It has been found on the lattice that, by decomposing the gauge fields into Abelian-monopole, Abelian-plain, and non-Abelian components, the removal of the monopoles does indeed lead to removal of the quark condensate [30,31].

In this paper, we address how chiral symmetry breaking and the generation of the nonzero quark condensate at $T<T_{c}$ can be explained in terms of this monopole model. The mechanism is based on the formation of bound states between quarks and monopoles. Like in condensed matter systems, in which "doping" of a material by atoms with an extra state leads to new set of states and alters its conductivity, the presence of monopoles radically affects the Dirac eigenvalue spectrum.

One obvious difficulty of the problem is the fact that a detailed understanding of the "lattice monopoles" is lacking; they are treated as effective objects whose parameters and behavior we can observe on the lattice and parametrize, but their microscopic structure has yet to be understood. In particular, the 't Hooft-Polyakov monopole solution includes a chiral-symmetry-breaking scalar field, while we know that, in massless QCD-like theories, chiral symmetry is locally unbroken. We assume that the zero modes in question are chiral themselves, like they are in the instantondyon theory, and that chiral symmetry breaking can only be achieved by a spontaneous breaking of the symmetry.

The other difficulty of the problem is the important distinction between fermionic zero modes of (i) monopoles and (ii) instanton-dyons. The latter include the so called $L$-type or twisted dyons, which possess 4-dimensional zero modes for antisymmetric fermions. Therefore, their collectivization naturally leads to chiral symmetry breaking, studied recently in Ref. [32], in a natural mechanism originally proposed for the instantons; for a review, see Ref. [33]. As follows from Banks-Casher relation [34], the quark condensate is proportional to density of Dirac eigenstates at zero eigenvalue.

The monopoles also have fermionic zero modes [35], which are 3-dimensional. They are, therefore, simply a bound state of a fermion and a monopole. In theories with extended supersymmetries, such objects do exist, fulfilling an important general requirement that monopoles need to come in particular supermultiplets, with fermionic spin $1 / 2$ for $\mathcal{N}=2$, or spins $1 / 2$ and 1 for $\mathcal{N}=4$. The antiperiodic boundary conditions for fermions in Matsubara time implies certain time dependence of the quark fields, and (as we will discuss in detail below) the lowest 4-dimensional Dirac eigenvalues produced by quarks bound to monopoles are the values $\lambda= \pm \pi T$, not at zero.

This, however, is only true for a single monopole. In a monopole ensemble with nonzero density, the monopolequark bound states are collectivized and Dirac eigenvalue spectrum is modified. The question is whether this effect can lead to a nonzero $\rho(\lambda=0) \propto\langle\bar{q} q\rangle$, and if so, whether it happens at the temperature at which chiral symmetry breaking is observed. As we will show below, we find affirmative answers to both these questions. The phenomenological monopole model parameters are such that a nonzero quark condensate is generated by monopoles at $T \approx T_{c}$.

As a final introductory comment, we note that our approach is to take as inputs the empirical monopole density $n(T)$ and ensemble of paths from our previous study. Using them, we calculate the corresponding Dirac eigenvalue spectrum. The backreaction of the fermions on monopole density and their motion is neglected. In this respect, our calculation is ideological similar to quenched lattice calculations, which also ignore quark backreaction on the gauge fields.

\section{FERMIONIC ZERO MODES OF SU(2) MONOPOLES}

In this section, we will overview the calculation of the fermion-monopole zero modes in the Georgi-Glashow model. First we will remind the reader of the result found by Jackiw and Rebbi [35] for the fermionic zero modes, and then go on to compute the matrix element between two monopoles in the basis of zero modes.

\section{A. Fermionic zero mode of a monopole}

This section is introductory, summarizing known results from Refs. [35,36] and presented for self-completeness of the paper. The Lagrangian of the Georgi-Glashow model (without fermions) is

$\mathcal{L}=-\frac{1}{4} F_{a}^{\mu \nu} F_{a \mu \nu}+\frac{1}{2}\left(D_{\mu} \phi\right)_{a}\left(D^{\mu} \phi\right)_{a}-\frac{1}{4} \lambda\left(\phi_{a} \phi_{a}-v^{2}\right)$

where

$$
F_{a}^{\mu \nu}=\partial^{\mu} A_{a}^{\nu}-\partial^{\nu} A_{a}^{\mu}+g \epsilon_{a b c} A_{b}^{\mu} A_{c}^{\nu},
$$

and

$$
\left(D^{\mu} \phi\right)_{a}=\partial^{\mu} \phi_{a}+g \epsilon_{a b c} A_{b}^{\mu} \phi_{c} .
$$


The fermionic part considered by Jackiw and Rebbi is

$$
\mathcal{L}_{\mathcal{F}}=i \bar{\psi}_{n} \gamma^{\mu}\left(D_{\mu} \psi\right)_{n}-G g \bar{\psi}_{n} \tau_{n m}^{a} \psi_{m} \phi_{a},
$$

with $G$ a constant, $\tau^{a}=\sigma^{a} / 2$, and

$$
\left(D_{\mu} \psi\right)_{n}=\partial^{\mu} \psi_{n}-i g \tau_{n m}^{a} A_{a}^{\mu} \psi_{m}
$$

The 't Hooft-Polyakov monopole solution has the form

$$
A_{a}^{0}=0, \quad A_{a}^{i}=\epsilon^{a i j} \hat{r}_{j} \frac{A(r)}{g}, \quad \phi_{a}=\hat{r}_{a} \frac{\phi(r)}{g} .
$$

With this ansatz, the equations of motion from the puregauge Lagrangian are

$$
\begin{aligned}
0= & \frac{2}{r^{2}} \frac{\mathrm{d}}{\mathrm{d} r}\left(r^{2} \frac{\mathrm{d} A}{\mathrm{~d} r}\right)-\frac{2}{r} \frac{\mathrm{d} A}{\mathrm{~d} r}+\frac{2}{r^{2}} \frac{\mathrm{d}}{\mathrm{d} r}(r A) \\
& -\frac{6}{r^{2}} A-\frac{6 g}{r} A^{2}-2 g^{2} A^{3}-\phi\left(\frac{2 g}{r}+2 g^{2} A\right), \\
0= & \frac{1}{r^{2}} \frac{\mathrm{d}}{\mathrm{d} r}\left(r^{2} \frac{\mathrm{d} \phi}{\mathrm{d} r}\right)-\frac{2}{r^{2}} \phi-\frac{4 g}{r} A \phi-2 g^{2} A^{2} \phi-2 U^{\prime}\left(|\phi|^{2}\right) \phi,
\end{aligned}
$$

and boundary conditions,

$$
\left.\left(r^{2} \frac{\mathrm{d} A}{\mathrm{~d} r}+2 r A\right)\right|_{r=0}=0,\left.\quad\left(r^{2} \frac{\mathrm{d} \phi}{\mathrm{d} r}\right)\right|_{r=0}=0 .
$$

This set of equations and boundary conditions, combined with single-valuedness of the fields at the origin give

$$
A(0)=\phi(0)=0,\left.\quad A(r)\right|_{r \rightarrow \infty}=-\frac{1}{r},\left.\quad \phi(r)\right|_{r \rightarrow \infty}=v,
$$

with $v$ a constant.

The Dirac equation for the fermion field is written in the form

$$
\begin{aligned}
& {\left[-i \vec{\alpha} \cdot \vec{\partial} \delta_{n m}+\frac{1}{2} A(r) \sigma_{n m}^{a}(\vec{\alpha} \times \overrightarrow{\hat{r}})_{a}+\frac{G \phi(r)}{2} \sigma_{n m}^{a} \hat{r}_{a} \beta\right] \psi_{m}} \\
& \quad=E \psi_{n}
\end{aligned}
$$

where $n, m=1,2$ are the isospin indices, $\sigma^{a}$ are the Pauli matrices, and

$$
\alpha_{i}=\left(\begin{array}{cc}
0 & \sigma_{i} \\
\sigma_{i} & 0
\end{array}\right), \quad \beta=-i\left(\begin{array}{cc}
0 & \mathbb{1} \\
-\mathbb{1} & 0
\end{array}\right) .
$$

For clarity, in Appendix we will discuss their representation of the Dirac matrices and the relation to fermion chirality.

In this representation of the Dirac matrices, the $\alpha_{i}, \beta$ are nondiagonal, and the only diagonal term is the energy. For a zero mode $E=0$, the problem is "chiral," in the sense that the 4-spinor $\psi$ splits into separate upper and lower components,

$$
\psi=\left(\begin{array}{c}
\psi^{+} \\
\psi^{-}
\end{array}\right)
$$

where $\psi_{l m}^{ \pm}$has four components, with $l$ corresponding to spin indices and $m$ corresponding to isospin (The SU(2) color is called isospin in the Georgi-Glashow model.) These upper and lower components can be further written as two scalar and vector fields,

$$
\psi_{l m}^{ \pm}=\left(g^{ \pm} \delta_{l m}+\vec{g}^{ \pm} \cdot \vec{\sigma}_{l n}\right) \sigma_{n m}^{2} .
$$

Carrying out the partial wave analysis (see Appendix of Ref. [35]) and finding the zero energy solution gives

$$
\begin{aligned}
& \vec{g}^{ \pm}(r)=0, \\
& g^{-}(r)=c^{-} \times \exp \left(\int_{0}^{r} \mathrm{~d} r^{\prime}\left[A\left(r^{\prime}\right)+\frac{1}{2} G \phi\left(r^{\prime}\right)\right]\right), \\
& g^{+}(r)=c^{+} \times \exp \left(\int_{0}^{r} \mathrm{~d} r^{\prime}\left[A\left(r^{\prime}\right)-\frac{1}{2} G \phi\left(r^{\prime}\right)\right]\right),
\end{aligned}
$$

The $g^{-}$solution is unnormalizable, so it is set to zero $\left(c^{-}=0\right)$. This gives the spinors

$$
\begin{aligned}
\psi_{l m}^{-}= & 0, \\
\psi_{l m}^{+}= & N \exp \left(\int_{0}^{r} \mathrm{~d} r^{\prime}\left[A\left(r^{\prime}\right)-\frac{1}{2} G \phi\left(r^{\prime}\right)\right]\right) \\
& \times\left(s_{l}^{+} s_{m}^{-}-s_{l}^{-} s_{m}^{+}\right),
\end{aligned}
$$

where $s^{ \pm}$are the eigenvectors of $\sigma^{3}$, and $N$ is a normalization.

The extension of the SU(2) 't Hooft-Polyakov monopole solution to $\mathrm{SU}(3)$ - with the same Georgi-Glashow-like Lagrangian - is discussed by A. Sinha [37]. In QCD, the $A_{0}$ field plays the role of the scalar (Higgs) field in the GeorgiGlashow model. For the purposes of this work, and for simplicity, we will study only the SU(2) case.

\section{B. Quark hopping matrix}

Recognizing fermionic binding to monopoles, we now proceed to description of their dynamics in the presence of ensembles of monopoles. The basis of the description is assumed to be the set of zero modes described in the previous section. The Dirac operator is written as a matrix in this basis, so that $i-j$ element is related to "hopping" between them. Such an approach originated from the "instanton liquid" model [33]. 
The matrix elements of the "hopping matrix"

$$
\mathbf{T}=\left(\begin{array}{cc}
0 & i T_{i j} \\
i T_{j i} & 0
\end{array}\right)
$$

where the $T_{i j} \mathrm{~s}$ are defined as the matrix element,

$$
T_{i j} \equiv\langle i|-i \not D| j\rangle,
$$

between the zero modes located on monopoles $i$ and antimonopoles $j$. In the $\mathrm{SU}(2)$ case we are considering, this is equivalent to

$$
\begin{aligned}
& T_{i j}=\left\langle\psi_{i} \mid x\right\rangle\langle x|-i \not D| y\rangle\left\langle y \mid \psi_{j}\right\rangle=\int \mathrm{d}^{3} x \psi_{k n}^{\dagger}\left(x-x_{i}\right)(-i \not D) \psi_{l m}\left(x-x_{j}\right) \\
&=\int \mathrm{d}^{3} x \psi_{k n}^{\dagger}\left(x-x_{i}\right)\left[-i(\vec{\alpha} \cdot \vec{\partial}+\vec{\alpha} \cdot \vec{\partial}-\vec{\alpha} \cdot \vec{\partial}) \delta_{n m}+\frac{1}{2}\left(A\left(x-x_{i}\right)+A\left(x-x_{j}\right)\right) \sigma_{n m}^{a}(\vec{\alpha} \times \overrightarrow{\hat{r}})_{a}\right. \\
&\left.+\frac{G\left(\phi\left(x-x_{i}\right)+\phi\left(x-x_{j}\right)\right)}{2} \sigma_{n m}^{a} \hat{r}_{a} \beta\right] \psi_{l m}\left(x-x_{j}\right) \\
&= \int \sum_{m} \mathrm{~d}^{3} x \psi_{k m}^{\dagger}\left(x-x_{i}\right)[-i \vec{\alpha} \cdot \vec{\partial}]^{k l} \psi_{l m}\left(x-x_{j}\right)
\end{aligned}
$$

where $\psi \mathrm{s}$ are zero modes with origin at $x_{i, j}$, the locations of the two monopoles, $n, m$ are the isospin/color indices, and we have used the fact that applying the Dirac operator to these wave functions gives zero.

The operator between the wave functions is

$$
\begin{aligned}
i \vec{\alpha} \cdot \vec{\partial}= & i\left(\begin{array}{cccc}
0 & 0 & 0 & 1 \\
0 & 0 & 1 & 0 \\
0 & 1 & 0 & 0 \\
1 & 0 & 0 & 0
\end{array}\right) \partial_{x}+i\left(\begin{array}{cccc}
0 & 0 & 0 & -i \\
0 & 0 & i & 0 \\
0 & -i & 0 & 0 \\
i & 0 & 0 & 0
\end{array}\right) \partial_{y} \\
& +i\left(\begin{array}{cccc}
0 & 0 & 1 & 0 \\
0 & 0 & 0 & -1 \\
1 & 0 & 0 & 0 \\
0 & -1 & 0 & 0
\end{array}\right) \partial_{z} .
\end{aligned}
$$

In the case where the original wave function only has an upper component, the resulting vector after applying this operator has only the lower component. Therefore, the matrix elements of the "hopping matrix" $T_{i j}$ are zero unless the fermionic zero modes have opposite chirality. So, a lefthanded fermion zero mode has nonzero overlap with a right-handed zero mode, and vice versa. To get the opposite chirality, we need to change the sign of the couplings. In the case of an $\mathrm{SU}(2)$ antimonopole, only the lower spinor survives, with the same wave function (the spin of the fermion flips). We will call these zero modes $\xi$.

This allows us to split the previous equation into two equations,

$$
\begin{aligned}
& T_{i j}=\int \mathrm{d}^{3} x \xi^{\dagger}\left(x-x_{i}\right)[-i \vec{\sigma} \cdot \vec{\partial}] \psi\left(x-x_{j}\right), \\
& T_{j i}=\int \mathrm{d}^{3} x \psi^{\dagger}\left(x-x_{i}\right)[-i \vec{\sigma} \cdot \vec{\partial}] \xi\left(x-x_{j}\right),
\end{aligned}
$$

where we use the appropriate one depending on whether $i(j)$ is a location of a monopole(antimonopole).

For the matrix element between a right-handed monopole zero mode and a left-handed antimonopole zero mode,

$$
T_{i j}=\int \mathrm{d}^{3} \vec{x} \sum_{m} \xi_{k m}^{\dagger}\left(\vec{x}-\vec{x}_{i}\right)[-i \vec{\sigma} \cdot \vec{\partial}]^{k l} \psi_{l m}\left(\vec{x}-\vec{x}_{j}\right),
$$

where $m$ is the traced-over isospin/color index, and $k, l$ are the spin indices (which are all convoluted). The operator is,

$$
[-i \vec{\sigma} \cdot \vec{\partial}]=\left(\begin{array}{cc}
-i \partial_{z} & -i \partial_{x}-\partial_{y} \\
-i \partial_{x}+\partial_{y} & i \partial_{z}
\end{array}\right) .
$$

For simplicity, we will treat each isospin/color case separately, so the only indices left are spin indices. We will denote the spatial-only part of the wave function (without spinors) with tildes.

(a) $m=1$ :

We will write $\xi_{k 1}$ as $\tilde{\xi} a_{k}$ and $\psi_{l 1}$ as $\tilde{\psi} a_{l}$. The wave function has spinors

$$
\begin{array}{r}
a_{i} \equiv s_{i}^{+} s_{1}^{-}-s_{i}^{-} s_{1}^{+}=\left(\begin{array}{c}
0 \\
-1
\end{array}\right), \\
a_{i}^{\dagger} \equiv s_{1}^{+} s_{i}^{-}-s_{1}^{-} s_{i}^{+}=\left(\begin{array}{ll}
1 & 0
\end{array}\right),
\end{array}
$$

and so

$$
\tilde{\xi} a_{k}^{\dagger}[-i \vec{\sigma} \cdot \vec{\partial}]^{k l} a_{l} \tilde{\psi}=\tilde{\xi}\left(i \partial_{x}+\partial_{y}\right) \tilde{\psi} .
$$

(b) $m=2$ :

The wave function has spinors

$$
\begin{aligned}
& b_{i} \equiv s_{i}^{+} s_{2}^{-}-s_{i}^{-} s_{2}^{+}=\left(\begin{array}{l}
1 \\
0
\end{array}\right), \\
& b_{i}^{\dagger} \equiv s_{2}^{+} s_{i}^{-}-s_{2}^{-} s_{i}^{+}=\left(\begin{array}{ll}
0 & -1
\end{array}\right),
\end{aligned}
$$


and so

$$
\tilde{\xi} b_{k}[-i \vec{\sigma} \cdot \vec{\partial}]^{k l} b_{l} \tilde{\psi}=\tilde{\xi}\left(i \partial_{x}-\partial_{y}\right) \tilde{\psi}
$$

Combining the two cases,

$$
\sum_{m} \xi_{k m}^{\dagger}[-i \vec{\sigma} \cdot \vec{\partial}]^{k l} \psi_{l m}=2 i \tilde{\xi} \partial_{x} \tilde{\psi}
$$

which then yields that,

$$
T_{i j}=2 i \int \mathrm{d}^{3} \vec{x} \tilde{\xi}\left(\vec{x}-\vec{x}_{i}\right) \partial_{x} \tilde{\psi}\left(\vec{x}-\vec{x}_{j}\right)
$$

For the matrix element between a left-handed antimonopole zero mode and a right-handed monopole zero mode, we get, similarly,

$$
T_{j i}=2 i \int \mathrm{d}^{3} \vec{x} \tilde{\psi}\left(\vec{x}-\vec{x}_{i}\right) \partial_{x} \tilde{\xi}\left(\vec{x}-\vec{x}_{j}\right)
$$

The full solutions to the equations of motion for $A(r)$ and $\phi(r)$ are well behaved at the origin, so we must use those in lieu of only considering asymptotics. The only analytic solution to the equations of motion is in the case where $\lambda=0$; this solution is known as the Bogomolnyi-PrasadSommerfeld (BPS) monopole [38,39]. In this case,

$$
H(\zeta)=\zeta \operatorname{coth}(\zeta)-1, \quad K(\zeta)=\frac{\zeta}{\sinh (\zeta)},
$$

where $\zeta=g v r$. In terms of these functions, our gauge fields are,

$A_{a}^{0}=0, \quad A_{a}^{i}=\epsilon^{a i j} \frac{r_{j}}{g r^{2}}(1-K(\zeta)), \quad \phi_{a}=\frac{r_{a}}{g r^{2}} H(\zeta)$.

This leads to the identification with our earlier notation,

$$
A(r)=\frac{1-K(g v r)}{r}, \quad \phi(r)=\frac{H(g v r)}{r} .
$$

For the monopole zero mode we get that, up to normalization,

$$
\tilde{\psi}=\frac{1}{2}(g v r)^{\frac{G}{2}+1} \operatorname{coth}\left(\frac{g v r}{2}\right) \sinh ^{-\frac{G}{2}}(g v r) .
$$

Then,

$$
\begin{aligned}
\partial_{x} \tilde{\psi}= & -\left(\frac{x}{4 r^{2}}\right)(g v r)^{\frac{G}{2}+1} \operatorname{coth}\left(\frac{1}{2} g v r\right) \sinh ^{-\frac{G}{2}-1}(g v r) \\
& \times(-(G+2) \sinh (g v r)+g G v r \cosh (g v r)+2 g v r),
\end{aligned}
$$

and similarly for the antimonopole zero mode wave function. Putting these solutions into the hopping matrix element equation for monopole-to-antimonopole, we get

$$
\begin{aligned}
T_{i j}\left(r_{0}\right)= & 2 i \int \mathrm{d}^{3} \vec{x} \tilde{\xi}\left(\left|r-r_{0}\right|\right) \partial_{x} \tilde{\psi}(r) \\
= & 2 i N^{2} \int \mathrm{d}^{3} \vec{x}\left(-\frac{x}{8 r^{2}}\right)(g v)^{G+2} r^{\frac{G+2}{2}}\left|r-r_{0}\right|^{\frac{G+2}{2}} \\
& \times \operatorname{coth}\left(\frac{1}{2} g v r\right) \sinh ^{-\frac{G}{2}-1}(g v r) \\
& \times \operatorname{coth}\left(\frac{1}{2} g v\left|r-r_{0}\right|\right) \sinh ^{-\frac{G}{2}}\left(g v\left|r-r_{0}\right|\right) \\
& \times(-(G+2) \sinh (g v r)+g G v r \cosh (g v r)+2 g v r) .
\end{aligned}
$$

The combination $g v r$ is dimensionless, as is $x / r$, so the integrand has dimension [energy] $(1 / r)$. The parameter $v$, in the BPS limit, is determined by the mass of the monopole, and $g$ is taken to be the same as in the strong coupling constant.

As an instructive exercise, taking $G, g, v=1$, we can evaluate this integral. The result is seen in Fig. 1. The first thing to note is that the result of integral is symmetric around the $x$-axis, and therefore only dependent on the combination $\left(y_{0}^{2}+z_{0}^{2}\right)$. So, for example, taking $r_{0}=$ $\left(x_{0}, 2,3\right)$ yields the same result as $r_{0}=\left(x_{0}, \sqrt{13}, 0\right)$, $r_{0}=\left(x_{0}, 3,2\right)$, etc. In addition, the function is odd under reflection from $x \rightarrow-x$. Therefore, we can evaluate the integral with $z_{0}=0, y_{0} \geq 0$, and $x_{0} \geq 0$, without loss of generality.

\section{DIRAC EIGENVALUES FOR MONOPOLE-ANTIMONOPOLE ENSEMBLES}

\section{A. The monopole ensembles}

We will use the monopole configurations studied in our previous path-integral simulation of monopole

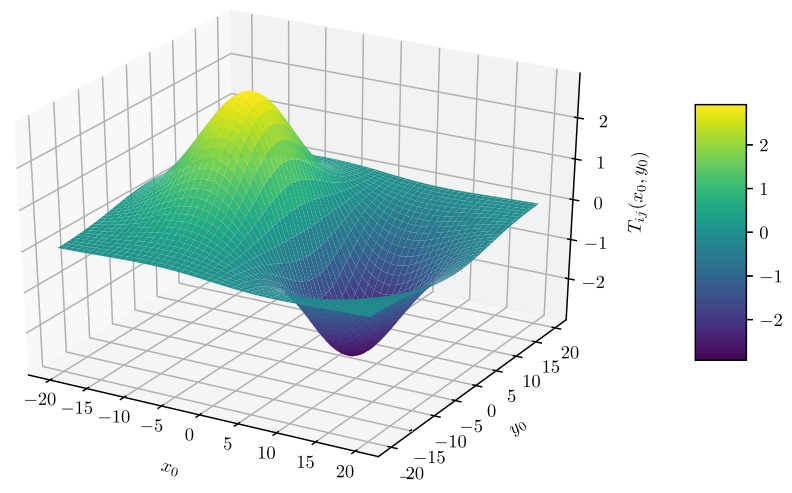

FIG. 1. Evaluation of $\Im\left(T_{i j}\left(r_{0}\right)\right)$ for $r_{0}$ in the $x y$ plane for $G=g=v=1$. 
Bose-Einstein condensation [19], and observe the effects of density and temperature on the eigenvalue spectrum of the Dirac operator for BPS monopoles. The configurations considered in Ref. [19] consisted of 8, 16, and 32 monopoles and anti-monopoles (Coulomb-interacting quantum particles with positive and negative charge) in a box with periodic boundary conditions. The particles are represented as paths in imaginary time, and Monte Carlo simulations are run to find the configurations with smallest Euclidean action. See Ref. [40] for a review of the pathintegral Monte Carlo method for studying many-body quantum systems. These configurations were simulated for a range of temperatures, and critical temperature for Bose-Einstein condensation, $T_{c}$, was obtained from studying both the permutation cycles of paths [18] and the finitesize scaling of the superfluid fraction of the systems [41]. The configurations of monopoles and anti-monopoles considered for this current study are a sampling of the path configurations generated in Ref. [19] for systems of 32 quantum Coulomb particles.

To constrain the values of $g$ and $v$, we can use the mass of the monopole. In the BPS limit, the mass is given by

$$
M=\frac{4 \pi v}{g} .
$$

The mass of the monopole was studied in Ref. [18] through lattice simulations of $\mathrm{SU}(2)$ pure-gauge theory, which shows a mass of around $2 T_{c}$ at $T=T_{c}$, and then rapidly grows as temperature increases. The density of monopoles in $\mathrm{SU}(2)$ gauge theories was studied in Ref. [15], and was parametrized by

$$
\frac{\rho}{T_{c}^{3}}\left(\frac{T}{T_{c}}\right)=\frac{0.557\left(T / T_{c}\right)^{3}}{\log \left(2.69\left(T / T_{c}\right)\right)^{2}} .
$$

\section{B. The quantization procedure}

We will evaluate the evolution matrix $U$, defined as timeordered integral of the hopping matrix in the previous section over the Matsubara periodic time $\tau \in[0, \beta]$. This matrix will then be diagonalized to find the eigenvalues for the fermion states. Because each eigenstate is still fermionic, each is required to fulfill the fermionic boundary conditions, namely that the state must return to minus itself after one rotation around the Matsubara circle. This defines quantization of the Dirac eigenvalues by,

$$
\lambda_{i}+\omega_{i, n}=\left(n+\frac{1}{2}\right) \frac{2 \pi}{\beta},
$$

where $\lambda_{i} \mathrm{~s}$ are the eigenvalues of the hopping matrix $\mathbf{T}$.

For monopoles that move in Euclidean time, we must integrate over the Matsubara circle to find the fermion frequencies,

$$
U=\oint_{\beta} d \tau e^{i H \tau}=-\mathbb{1}
$$

This can be approximated by

$$
\begin{aligned}
-\mathbb{1} & \approx e^{i H_{m} \Delta \tau} \ldots e^{i H_{2} \Delta \tau} e^{i H_{1} \Delta \tau} \\
& \approx\left(1+i H_{m} \Delta \tau-\ldots\right) \ldots\left(1+i H_{1} \Delta \tau-\ldots\right)
\end{aligned}
$$

for $m$ time slices. We diagonalize the resulting matrix on the right-hand side and solve to find the quantity $\lambda+\omega$.

\section{Dirac eigenvalue spectra and chiral symmetry breaking}

For simplicity, we will work in units of $T_{c}$ (i.e., $T_{c}=1$ ) when doing this calculation (for mass and temperature, for example), and units of length will be defined by the density of monopoles $r \sim \rho^{-1 / 3}$ in units of $1 / T_{c}$.

Before we begin, to estimate what the effects of temperature will be on our results, we can evaluate the integral in Eq. (25) with different values of the parameters, corresponding to the range of temperatures we will simulate. We will take $G=g=\sqrt{4 \pi / 3}$ - as would be the case in QCD—and $v$ from the monopole mass, taken from Ref. [18]. The former assumption comes from the idea that the Higgs field and the three gauge fields in the GeorgiGlashow model correspond to the $A^{\mu}$ in $\mathrm{QCD}$, which all have the same value for the coupling, and nominally taking $\alpha_{s}=g^{2} /(4 \pi)=1 / 3$. In the BPS limit, the mass of the monopole is related to the coupling by $M=4 \pi v / g$.

The results for $T / T_{c}=1,1.05,1.1$, and 1.2 are seen in Fig. 2(a), (b), (c), and (d), respectively. Compared to the $g=G=v=1$ case, the range of the zero-mode hopping is significantly smaller- $\sim 10$ units of length in $x_{0}$ to a peak for the former case and 2.5 for $T=T_{c}$-and decreases rapidly with temperature. In addition, the peaks of the function reduce in magnitude and very quickly become much sharper as temperature is increased. Therefore, the contribution to the $T_{i j}$ matrix at temperatures above $T_{c}$ will only come from "local" hopping (i.e., only when there is a monopole-antimonopole molecular bound state), while at $T_{c}$, the ensemble contributes and there can be a chain of hopping. We note that taking $G=g=\sqrt{2 \pi / 3}$ or $G=$ $g=\sqrt{2 \pi}$ (and the corresponding masses of the monopoles) does not change this behavior, and therefore does not change the results of the study.

To make this explicit, we construct the hopping matrix, as described above, for $\mathrm{SU}(2)$ monopole configurations at $T / T_{c}=1,1.05,1.1,1.2$ and 1.5, and calculate the eigenvalue spectra of the Dirac operator at each of those temperatures. For each of the temperatures, we take 400 path configurations of 32 time slices, each with 32 particles in a box with periodic boundary conditions - the box is repeated to the extent necessary to study the whole range of $x_{0}, y_{0}, z_{0}$. At each time slice, the hopping matrix is 


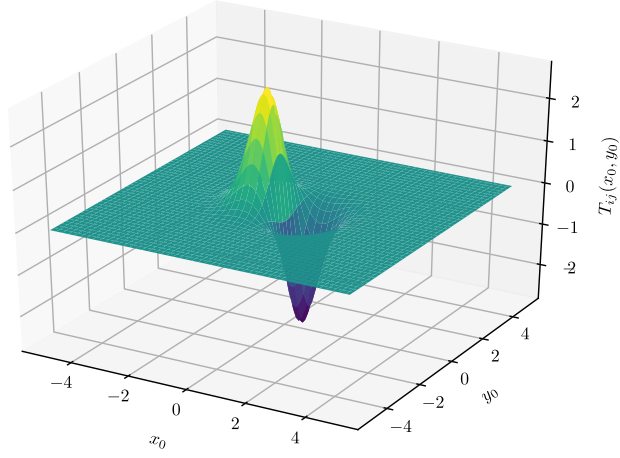

(a) $T=1 T_{c}$

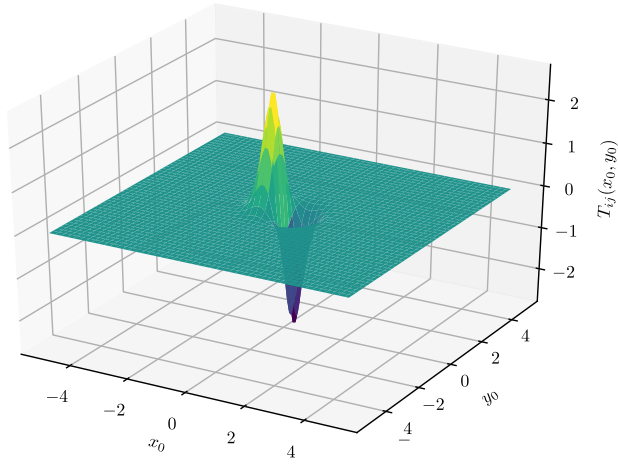

(c) $T=1.1 T_{c}$

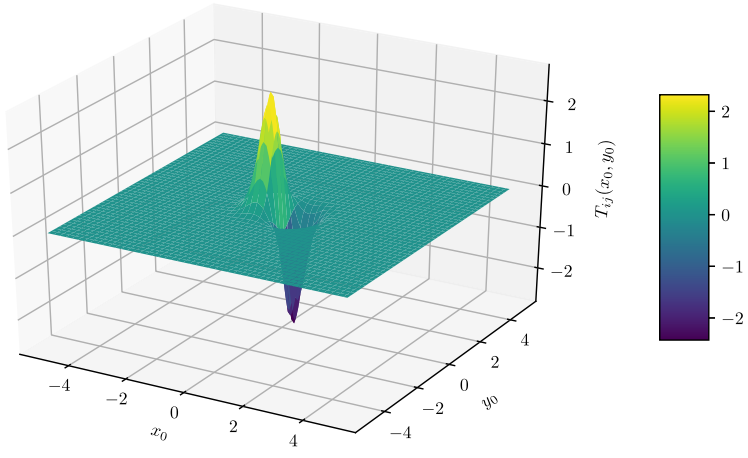

(b) $T=1.05 T_{c}$

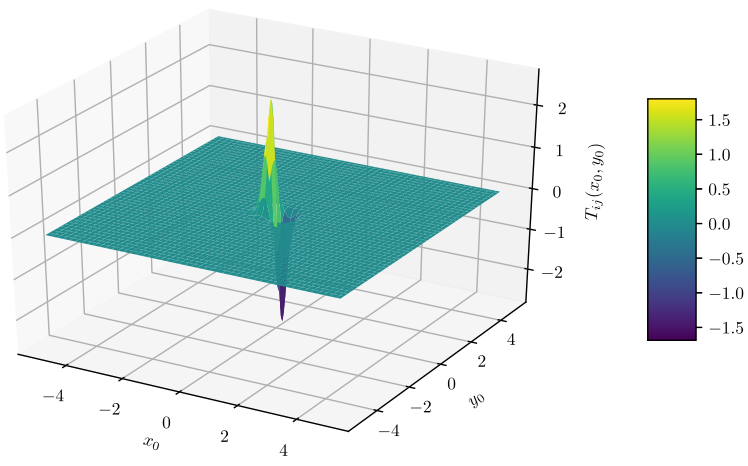

(d) $T=1.2 T_{c}$

FIG. 2. Evaluation of $\Im\left(T_{i j}\left(r_{0}\right)\right)$ for $r_{0}$ in the $x y$ plane for different temperatures.

calculated; the matrices are then path-exponentiated to get the hopping matrix for the ensemble configuration.

The spectral density of eigenvalues is given by

$$
\rho(\lambda)=\frac{1}{V} \sum_{i} \delta\left(\lambda-\lambda_{i}\right)
$$

The eigenvalues of the configurations-before imposing the fermion boundary conditions-are seen in Fig. 3. We can then compute the eigenvalues of the Dirac operator with

$$
\omega_{i, n}=\left(n+\frac{1}{2}\right) \frac{2 \pi}{\beta}-\lambda_{i}
$$

Considering only the $n= \pm 1$ case, so that $\omega_{i}= \pm \pi T-\lambda_{i}$, we get the distributions shown in Fig. 4(a), (b), (c), and (d) for $T / T_{c}=1,1.05,1.1$, and 1.2 , respectively. We note that this procedure was also repeated for systems of 16,20 , and 24 particles, and yielded the same results as those above.

The Banks-Casher relation [34] makes the connection between the density of eigenvalues at $\omega=0$ and the magnitude of the chiral condensate. In studying the monopole contribution to the chiral condensate, it is important to note that we can only approach the critical temperature $T_{c}$ from above, as we do not have any lattice data on monopole density, mass, or correlations below $T_{c}$. As a result, we will primarily focus on the gap in eigenvalue spectrum around $\omega=0$ as a proxy for the chiral transition.

The first, and most important, thing to notice is that when $T=T_{c}$, the eigenvalue distribution has a finite density at

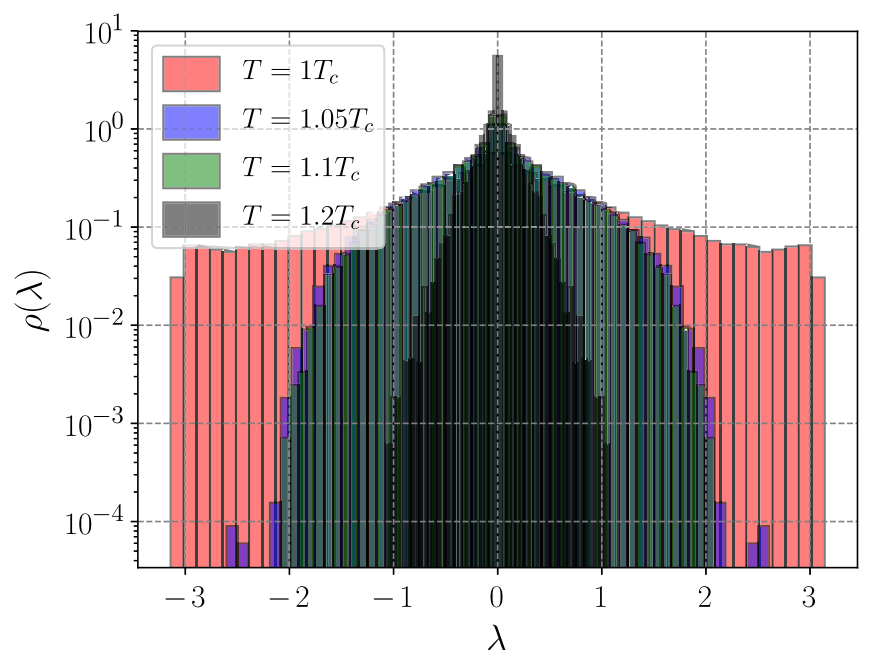

FIG. 3. Eigenvalue distribution for $T / T_{c}=1$ (red), 1.1 (blue), and 1.2 (green). Note the logarithmic scale. 


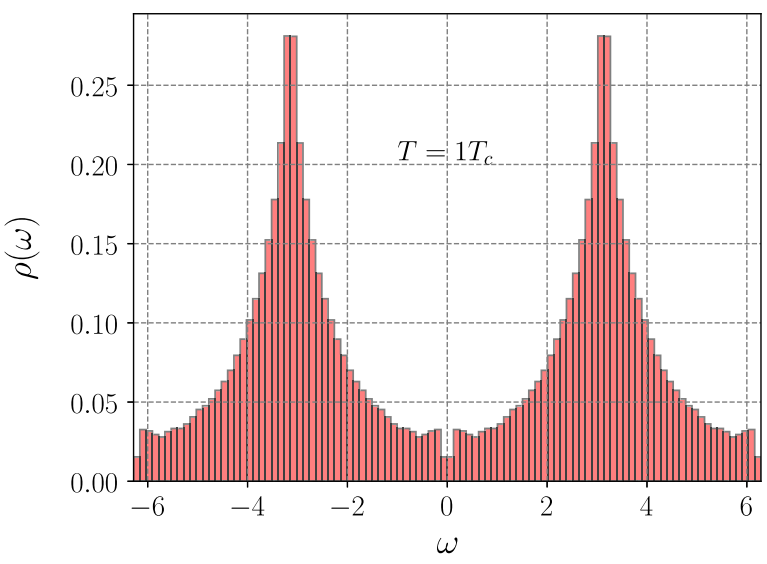

(a) $T=1 T_{c}$

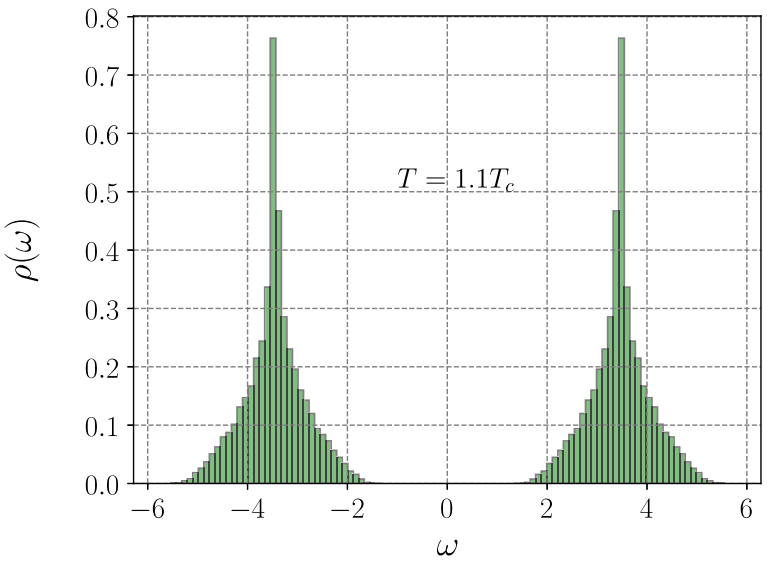

(c) $T=1.1 T_{c}$

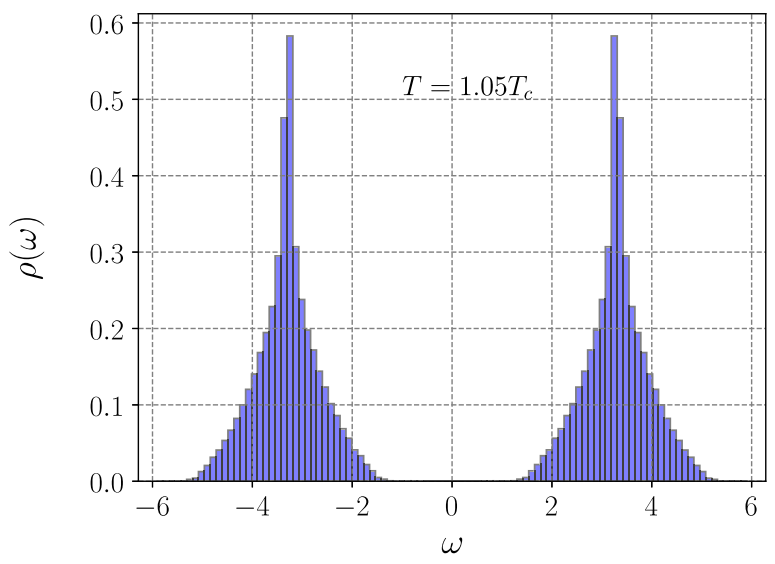

(b) $T=1.05 T_{c}$

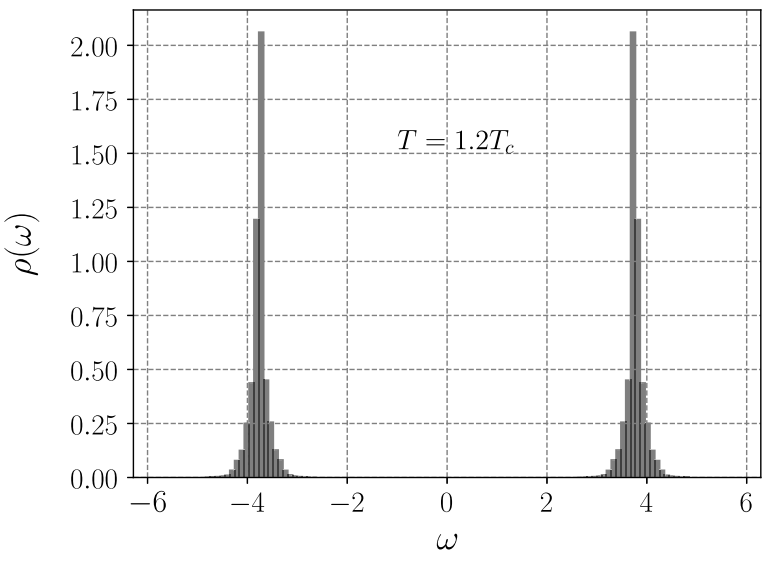

(d) $T=1.2 T_{c}$

FIG. 4. Distributions of Dirac eigenvalues for $T / T_{c}=$ (a) 1 , (b) 1.05 , (c) 1.1 , and (d) 1.2, respectively.

$\omega=0$ [see Fig. 4(a)], which indicates the nonzero value of the chiral condensate; there is no gap in the spectrum present at $T=T_{c}$. (A small dip seen around zero is a consequence of finite size of the system, well known and studied on the lattice and in topological models. It should be essentially ignored in extrapolation to zero.)

Furthermore, one can see the onset of nonzero density at zero eigenvalue by looking at the smallest eigenvalue in each configuration. Figure 5 shows the mean smallest eigenvalue as a function of temperature. We fit the data with the function

$$
\left\langle\omega_{\min }\right\rangle=A\left(T-T_{c}\right)^{\nu},
$$

where $\langle\ldots\rangle$ indicates an average over configurations. The fit parameters were found to be $A=6.29$ and $\nu=.60$. We note that this exponent $\nu$ is compatible with the critical exponent found from the diverging correlation length at the deconfinement temperature, which for the $3 \mathrm{~d}$ Ising model is $\nu \approx .63$, and is also consistent with the monopole BEC

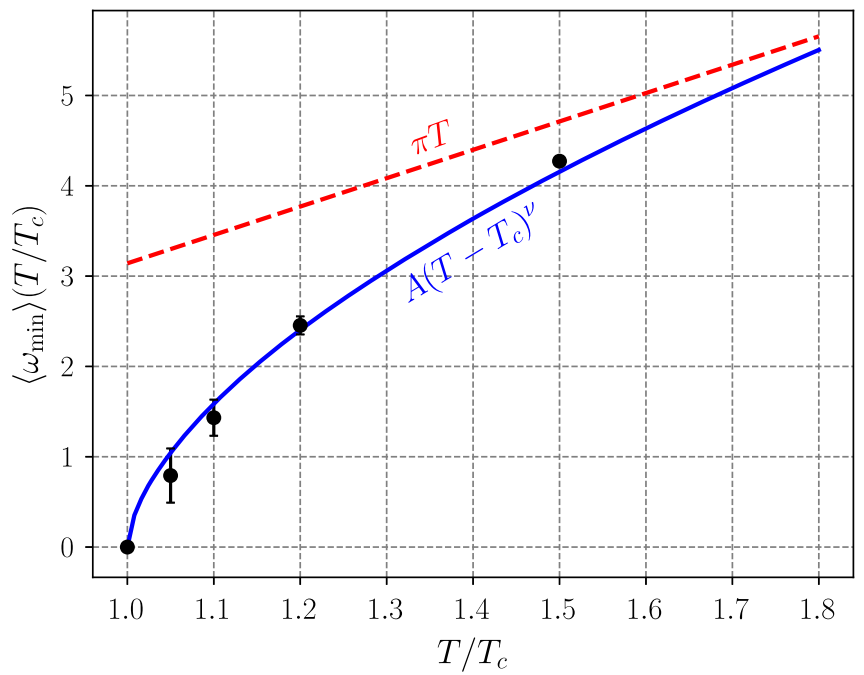

FIG. 5. Minimum Dirac eigenvalue as a function of temperature. The black dots are values from our simulations, the blue line is the fit $A\left(T-T_{c}\right)^{\nu}$, and the red dashed line is $\pi T$. 
transition found in Ref. [18]. For different values of $G, g$, the scaling exponent stays approximately the same $(\nu \approx 0.58$ to 0.61$)$. Previous studies have shown that the deconfining phase transition of the Georgi-Glashow model falls in the Ising universality class $[42,43]$; our result is another confirmation of this property of the model.

\section{SUMMARY}

In qualitative terms, the mechanism of chiral symmetry breaking based on monopoles is as follows. A single monopole (or anti-monopole) generates additional quark and antiquark bound states. At high temperatures, the monopoles have large mass and the probability of hopping is therefore low. The 4d Dirac operator eigenvalues are well localized near the fermionic Matsubara frequencies $2 \pi T(n+1 / 2)$. Using the condensed matter analogy, one may say that a matter is an insulator.

However, as $T$ decreases toward $T_{c}$, the amplitudes of quark "hopping" from one monopole to an antimonopole (and vice versa) grow. Eventually, at some critical density, quarks become "collectivized" and are able to travel very far from their original locations. The physics of the mechanism is similar to insulator-metal transition in condensed matter under pressure.

Technically, the central point is the distinction between the evolution operator and quantization of the fermionic states on one hand, and the Dirac operator and its eigenvalues.

Quantitatively, we found that not only the mechanism works in principle, but that a noticeable quark condensate does appear at $T \approx T_{c}$, practically simultaneously with the deconfinement phase transition, seen by the BEC of monopoles. This observation is consistent with what has been observed in quenched lattice calculations.

Finally, let us comment on the dependence of chiral symmetry breaking on the fermion periodicity phase. We have not studied it in this work, but note that for periodic quarks, the Matsubara frequencies shift to bosonic set $2 \pi T n$, including $n=0$. Therefore the monopoles would produce a nonzero quark condensate at any density. This comment implies that the chiral transition is in general some function of the periodicity phase, and its coincidence with deconfinement only happens for the antiperiodic quarks we studied.

\section{ACKNOWLEDGMENTS}

The authors thank the Institute for Advanced Computational Science (IACS) at Stony Brook University for the use of its LI-red computational cluster. This work was supported in part by the U.S. D.O.E. Office of Science, under Contract No. DE-FG-88ER40388.

\section{APPENDIX: GAMMA MATRICES AND CHIRALITY OF THE MONOPOLE ZERO MODES}

The representation of the Dirac matrices used by Jackiw and Rebbi and mentioned in the text correspond to the definition

$$
\gamma_{4}=\beta, \quad \gamma_{4} \vec{\gamma}=-i \vec{\alpha},
$$

with the representation of the gamma matrices

$$
\gamma_{4}=-i\left(\begin{array}{cc}
0 & \mathbb{1} \\
-\mathbb{1} & 0
\end{array}\right), \quad \gamma_{i}=\left(\begin{array}{cc}
-\sigma_{i} & 0 \\
0 & \sigma_{i}
\end{array}\right), \quad \gamma_{5}=i\left(\begin{array}{ll}
0 & \mathbb{1} \\
\mathbb{1} & 0
\end{array}\right) .
$$

Note that this form is different both from the standard Dirac representation

$\gamma_{0}=\left(\begin{array}{cc}\mathbb{1} & 0 \\ 0 & -\mathbb{1}\end{array}\right), \quad \gamma_{i}=\left(\begin{array}{cc}0 & \sigma_{i} \\ -\sigma_{i} & 0\end{array}\right), \quad \gamma_{5}=\left(\begin{array}{ll}0 & \mathbb{1} \\ \mathbb{1} & 0\end{array}\right)$,

and the Weyl one, in which

$$
\gamma_{0}=\left(\begin{array}{ll}
0 & \mathbb{1} \\
\mathbb{1} & 0
\end{array}\right), \quad \gamma_{i}=\left(\begin{array}{cc}
0 & \sigma_{i} \\
-\sigma_{i} & 0
\end{array}\right), \quad \gamma_{5}=\left(\begin{array}{cc}
-\mathbb{1} & 0 \\
0 & \mathbb{1}
\end{array}\right) .
$$

Standard definition of fermion chirality (left and right polarizations) is related to projectors $\left(1 \pm \gamma_{5}\right) / 2$, and so only the last Weyl representation, in which $\gamma_{5}$ is diagonal, is really chiral. The zero modes of pure gauge solitons, such as instantons and instanton-dyons, are chiral in this standard sense.

The 2-component zero modes found by Jackiw and Rebbi are often called "chiral" in literature, but they are not chiral in the standard sense, as seen already from the fact that in their representation $\gamma_{5}$ is not diagonal. Furthermore, as seen directly from the Lagrangian of the GeorgiGlashow model, fermions interact with a scalar field, and this vertex mixes the left and right polarizations, explicitly breaking chiral symmetry.

In pure gauge theory with massless fermions (which we discuss), the $\mathrm{SU}\left(N_{f}\right)$ chiral symmetry is exact. So, when the lattice monopoles-whatever their microscopic structure may be-have fermionic bound states, those should belong to the representation of the standard chiral symmetry, rather than the one with the quotation marks. So, while we use the 't Hooft-Polyakov monopole and its Jackiw-Rebbi zero modes as an example, we do not expect it correctly reproduce their chiral properties. We assume that the zero modes of monopoles in gauge theories without scalars (such as QCD) are truly chiral in the usual sense. 
[1] P. A. M. Dirac, Quantised singularities in the electromagnetic field, Proc. R. Soc. A 133, 60 (1931).

[2] G. 't Hooft, Magnetic monopoles in unified gauge theories, Nucl. Phys. B79, 276 (1974).

[3] A. M. Polyakov, Particle spectrum in the quantum field theory, Pis'ma Zh. Eksp. Teor. Fiz. 20, 430 (1974) [JETP Lett. 20, 194 (1974)].

[4] E. Shuryak, Instanton-dyon ensembles reproduce deconfinement and chiral restoration phase transitions, arXiv: 1710.03611.

[5] N. Dorey and A. Parnachev, Instantons, compactification and S duality in $N=4$ SUSY Yang-Mills theory. 2, J. High Energy Phys. 08 (2001) 059.

[6] E. Poppitz and M. Unsal, Seiberg-Witten and 'Polyakovlike' magnetic bion confinements are continuously connected, J. High Energy Phys. 07 (2011) 082.

[7] E. Poppitz, T. Schäfer, and M. Unsal, Continuity, Deconfinement, and (Super) Yang-Mills Theory, J. High Energy Phys. 10 (2012) 115.

[8] Y. Nambu, Strings, Monopoles and gauge fields, Phys. Rev. D 10, 4262 (1974).

[9] G. 't Hooft, Topology of the gauge condition and new confinement phases in nonabelian gauge theories, Nucl. Phys. B190, 455 (1981).

[10] S. Mandelstam, Vortices and quark confinement in nonabelian gauge theories, Phys. Rep. 23, 245 (1976).

[11] M. L. Laursen and G. Schierholz, Evidence for monopoles in the quantized SU(2) lattice vacuum: A study at finite temperature, Z. Phys. C 38, 501 (1988).

[12] Y. Koma, M. Koma, E. M. Ilgenfritz, T. Suzuki, and M. I. Polikarpov, Duality of gauge field singularities and the structure of the flux tube in Abelian projected SU(2) gauge theory and the dual Abelian Higgs model, Phys. Rev. D 68 , 094018 (2003).

[13] V. G. Bornyakov et al. (DIK Collaboration), Dynamics of monopoles and flux tubes in two flavor dynamical QCD, Phys. Rev. D 70, 074511 (2004).

[14] T. Suzuki, M. Hasegawa, K. Ishiguro, Y. Koma, and T. Sekido, Gauge invariance of color confinement due to the dual Meissner effect caused by Abelian monopoles, Phys. Rev. D 80, 054504 (2009).

[15] A. D'Alessandro and M. D'Elia, Magnetic monopoles in the high temperature phase of Yang-Mills theories, Nucl. Phys. B799, 241 (2008).

[16] C. Bonati and M. D'Elia, The maximal Abelian gauge in SU(N) gauge theories and thermal monopoles for $N=3$, Nucl. Phys. B877, 233 (2013).

[17] J. Liao and E. Shuryak, Magnetic Component of QuarkGluon Plasma is also a Liquid, Phys. Rev. Lett. 101, 162302 (2008).

[18] A. D'Alessandro, M. D'Elia, and E. V. Shuryak, Thermal monopole condensation and confinement in finite temperature Yang-Mills theories, Phys. Rev. D 81, 094501 (2010).

[19] A. Ramamurti and E. Shuryak, Effective model of QCD magnetic monopoles from numerical study of one- and twocomponent coulomb quantum Bose gases, Phys. Rev. D 95, 076019 (2017).
[20] C. Bonati, A. Di Giacomo, L. Lepori, and F. Pucci, Monopoles, abelian projection and gauge invariance, Phys. Rev. D 81, 085022 (2010).

[21] C. Bonati, A. Di Giacomo, and M. D'Elia, Detecting monopoles on the lattice, Phys. Rev. D 82, 094509 (2010).

[22] A. Di Giacomo, QCD monopoles, abelian projections and gauge invariance, arXiv:1707.07896.

[23] J. Liao and E. Shuryak, Strongly coupled plasma with electric and magnetic charges, Phys. Rev. C 75, 054907 (2007).

[24] J. Liao and E. Shuryak, Electric flux tube in magnetic plasma, Phys. Rev. C 77, 064905 (2008).

[25] C. Ratti and E. Shuryak, The role of monopoles in a gluon plasma, Phys. Rev. D 80, 034004 (2009).

[26] J. Xu, J. Liao, and M. Gyulassy, Bridging soft-hard transport properties of quark-Gluon plasmas with CUJET3.0, J. High Energy Phys. 02 (2016) 169.

[27] J. Xu, J. Liao, and M. Gyulassy, Consistency of perfect fluidity and jet quenching in semi-quark-gluon monopole plasmas, Chin. Phys. Lett. 32, 092501 (2015).

[28] A. Ramamurti and E. Shuryak, The role of QCD monopoles in jet quenching, Phys. Rev. D 97, 016010 (2018).

[29] P. Cea, L. Cosmai, F. Cuteri, and A. Papa, Flux tubes in the QCD vacuum, Phys. Rev. D 95, 114511 (2017).

[30] T. Suzuki and I. Yotsuyanagi, A possible evidence for Abelian dominance in quark confinement, Phys. Rev. D 42, 4257 (1990).

[31] O. Miyamura, Chiral symmetry breaking in gauge fields dominated by monopoles on SU(2) lattices, Phys. Lett. B 353, 91 (1995).

[32] R. Larsen and E. Shuryak, Instanton-dyon ensemble with two dynamical quarks: The chiral symmetry breaking, Phys. Rev. D 93, 054029 (2016).

[33] T. Schäfer and E. V. Shuryak, Instantons in QCD, Rev. Mod. Phys. 70, 323 (1998).

[34] T. Banks and A. Casher, Chiral symmetry breaking in confining theories, Nucl. Phys. B169, 103 (1980).

[35] R. Jackiw and C. Rebbi, Solitons with fermion number $1 / 2$, Phys. Rev. D 13, 3398 (1976).

[36] C. J. Callias, Spectra of fermions in monopole fields: Exactly soluble models, Phys. Rev. D 16, 3068 (1977).

[37] A. Sinha, SU(3) Magnetic monopoles, Phys. Rev. D 14, 2016 (1976).

[38] E. B. Bogomolny, Stability of classical solutions, Yad. Fiz. 24, 861 (1976) [Sov. J. Nucl. Phys. 24, 449 (1976)].

[39] M. K. Prasad and C. M. Sommerfield, An Exact Classical Solution for the 't Hooft Monopole and the Julia-Zee Dyon, Phys. Rev. Lett. 35, 760 (1975).

[40] D. M. Ceperley, Path integrals in the theory of condensed helium, Rev. Mod. Phys. 67, 279 (1995).

[41] E. L. Pollock and K. J. Runge, Finite-size-scaling analysis of a simulation of the ${ }^{4} \mathrm{He}$ superfluid transition Phys. Rev. B 46, 3535 (1992).

[42] G. V. Dunne, I. I. Kogan, A. Kovner, and B. Tekin, Deconfining phase transition in $2+1 D$ : the Georgi-Glashow model J. High Energy Phys. 01 (2001) 032.

[43] Y. V. Kovchegov and D. T. Son, Critical Temperature of the Deconfining Phase Transition in $(2+1) d$ Georgi-Glashow Model J. High Energy Phys. 01 (2003) 050. 